Constr. Approx. (2003) 19: 373-397

DOI: $10.1007 / \mathrm{s} 00365-002-0515-0$

CONSTRUCTIVE APPROXIMATION

\title{
Self-Affine Sets and Graph-Directed Systems
}

\author{
Xing-Gang He, Ka-Sing Lau, and Hui Rao
}

\begin{abstract}
A self-affine set in $\mathbb{R}^{n}$ is a compact set $T$ with $A(T)=\bigcup_{d \in \mathcal{D}}(T+d)$ where $A$ is an expanding $n \times n$ matrix with integer entries and $\mathcal{D}=\left\{d_{1}, d_{2}, \ldots, d_{N}\right\} \subset \mathbf{Z}^{n}$ is an $N$-digit set. For the case $N=|\operatorname{det}(A)|$ the set $T$ has been studied in great detail in the context of self-affine tiles. Our main interest in this paper is to consider the case $N>|\operatorname{det}(A)|$, but the theorems and proofs apply to all the $N$. The self-affine sets arise naturally in fractal geometry and, moreover, they are the support of the scaling functions in wavelet theory. The main difficulty in studying such sets is that the pieces $T+d, d \in \mathcal{D}$, overlap and it is harder to trace the iteration. For this we construct a new graph-directed system to determine whether such a set $T$ will have a nonvoid interior, and to use the system to calculate the dimension of $T$ or its boundary (if $T^{o} \neq \emptyset$ ). By using this setup we also show that the Lebesgue measure of such $T$ is a rational number, in contrast to the case where, for a self-affine tile, it is an integer.
\end{abstract}

\section{Introduction}

Let $M_{n}(\mathbb{Z})$ denote the set of $n \times n$ matrices with integer entries. A matrix $A \in M_{n}(\mathbb{Z})$ is called expanding if all its eigenvalues have moduli $>1$. Throughout this paper we assume that $A$ is an expanding matrix and that $\mathcal{D}=\left\{d_{1}, \ldots, d_{N}\right\} \subseteq \mathbb{Z}^{n}$ is a set of $N$ distinct vectors, called a digit set. Let

$$
\varphi_{i}(x)=A^{-1}\left(x+d_{i}\right), \quad 1 \leq i \leq N .
$$

The family $\left\{\varphi_{i}(x)\right\}_{i=1}^{N}$ is called an iterated function system (IFS). It is known that under a suitable norm in $\mathbb{R}^{n}$ the maps $\varphi_{i}(x), 1 \leq i \leq N$, are contractive [19, p. 29]. Therefore, there is a unique nonempty compact set $T=T(A, \mathcal{D})$ satisfying

$$
T=\bigcup_{i=1}^{N} \varphi_{i}(T)
$$

[8]. An equivalent form of the above equation is

$$
A(T)=\bigcup_{i=1}^{N}\left(T+d_{i}\right)=T+\mathcal{D} .
$$

Date received: February 28, 2001. Date revised: November 28, 2001. Date accepted: April 3, 2002. Communicated by Rong-Qing Jia. Online publication: January 10, 2003.

AMS classification: Primary, 52C20, 52C22; Secondary, 28A80.

Key words and phrases: Attractor, Boundary, Contraction, Iterated function system, Hausdorff dimension, Graph-directed construction, Overlapping, Tiles, Self-affine sets, Self-similar sets. 
Also $T$ can be expressed in radix expansions: $T=\left\{\sum_{k=1}^{\infty} A^{-k} d_{k}: d_{k} \in \mathcal{D}\right\}$. We call $T$ a self-affine set, and a self-affine region, if it has positive Lebesgue measure. If in addition $A$ is a constant multiple of an orthonormal matrix (i.e., $A$ is a similarity and the $\left\{\varphi_{i}\right\}_{i=1}^{N}$ are similitudes), then we call $T$ a self-similar set or a self-similar region instead.

It is easy to see that if $N<|\operatorname{det}(A)|$, then $T$ has zero Lebesgue measure. If $N=$ $|\operatorname{det}(A)|$, then $T$ can have positive or zero Lebesgue measure, and in the first case it is equivalent to $T^{o} \neq \varnothing[19]$. We call such $T$ a self-affine tile. The geometric and algebraic properties of the self-affine tiles have been studied in great detail in literature recently (see, e.g., [1], [7], [12], [16], [18], [19], [20], [21], [30], [32]). The case for $N \geq|\operatorname{det}(A)|$ arises naturally in the study of iterated function systems with overlaps [23], [28]. The corresponding self-affine regions also appear in wavelet theory, as they are the supports of the important class of scaling functions [5]:

$$
f(x)=\sum_{d_{i} \in D} w_{i} f\left(A x+d_{i}\right) .
$$

The solution $f$ for $A=2$ and $\mathcal{D}=\{0,1, \ldots, N\}$ has been studied in great detail. The general cases on $\mathbb{R}^{n}$ have been sketched briefly in [4, Appendix] and detailed investigations were given only for special cases. Note that $T$ is a self-affine region is necessary for the existence of the $L^{1}$-solution. So far there is little attention focused directly on the class of self-affine regions and that is the main purpose of our investigation in this paper.

If $T$ is a self-affine tile, then its copies $\left\{\varphi_{i}(T)\right\}$ are essentially disjoint (i.e., the intersection of any two copies has zero Lebesgue measure). This property plays a key role in the investigation of self-affine tiles. On the other hand, it is easy to see that for a selfaffine set with $N>|\operatorname{det}(A)|$ (also for the case of $N \leq|\operatorname{det}(A)|$ and the IFS $\left\{\varphi_{i}\right\}_{i=1}^{N} \operatorname{does}$ not satisfy the open set condition [8]), its copies have overlaps (not essentially disjoint). The overlaps create much complication, as described by Odlyzko [27, p. 213]: "the task of classifying them seems hopeless."

A systematic attempt to study the self-affine region is in [13] where some fundamental properties have been established. Analogous to the self-affine tiles [19], it was proved that

Theorem 1.1. Let $A$ be an expanding matrix in $M_{n}(\mathbb{Z})$ and let $\mathcal{D} \subseteq \mathbb{Z}^{n}$ be a digit set with $N \geq|\operatorname{det} A|$, then $T$ is a self-affine region if and only if $T^{o} \neq \emptyset$. In this case $T=\bar{T}^{o}$ and the boundary $\partial T$ of $T$ has Lebesgue measure zero.

In this paper we will consider the following questions:

(1) How to determine that $T$ has a nonvoid interior constructively based on $A$ and $\mathcal{D}$ ?

(2) What is the Lebesgue measure for such a self-affine region? It is known that the Lebesgue measure of a self-affine tile is an integer [20].

(3) How to compute the Hausdorff dimension of $T$ or its boundary when it is a self-affine (self-similar) region?

We will introduce a graph-directed system to investigate these questions. Since $T$ ( $A$, $\left.\mathcal{D}-x_{0}\right)=T(A, \mathcal{D})-(A-E)^{-1} x_{0}$ (where $E$ is the unit matrix) [18], we always assume that $d_{1}=0$. First, we select a digit set $C \in \mathbb{Z}^{n}$ and form an auxiliary self-affine tile 
$\Gamma=T(A, \mathcal{C})$ such that

$$
T \subseteq \Gamma^{o} \quad \text { and } \quad \Gamma+\mathcal{D} \subseteq A \Gamma .
$$

The associated auxiliary IFS is

$$
\psi_{j}(x)=A^{-1}\left(x+c_{j}\right), \quad j=1, \ldots, q,
$$

where $q:=|\operatorname{det}(A)|$. Let $\Sigma_{q}^{k}=\left\{J=\left(j_{1}, \ldots, j_{k}\right): 1 \leq j_{s} \leq q\right\}, \Sigma_{q}=\bigcup_{k=1}^{\infty} \Sigma_{q}^{k}$ and

$$
c_{J}=c_{j_{k}}+A c_{j_{k-1}}+\cdots+A^{k-1} c_{j_{1}} .
$$

Observing that, for each $k,\left\{\psi_{J}(\Gamma)=A^{-k}\left(\Gamma+c_{J}\right): J \in \Sigma_{q}^{k}\right\}$ is a partition of $\Gamma$ and the diameter of $\psi_{J}(\Gamma), J \in \Sigma_{q}^{k}$, tends to zero when $k$ tends to infinity. The key of this auxiliary system is that we can rewrite

$$
T=\bigcap_{k=1}^{\infty} \bigcup_{I \in \Sigma_{N}^{k}} \varphi_{I}(\Gamma)
$$

as

$$
T=\bigcap_{k=1}^{\infty} \bigcup_{J \in \mathcal{S}_{k}} \psi_{J}(\Gamma)
$$

where $\mathcal{S}_{k}=\left\{J \in \Sigma_{q}^{k}: \psi_{J}(\Gamma) \cap \bigcup_{I \in \Sigma_{N}^{k}} \varphi_{I}(\Gamma) \neq \emptyset\right\}$. Note that $\left\{\varphi_{I}(\Gamma): I \in \Sigma_{N}^{k}\right\}$ in the first expression is an overlapping family, but $\left\{\psi_{J}(\Gamma): J \in \Sigma_{q}^{k}\right\}$ in the second expression is a nonoverlapping family which gives an advantage in handling the self-affine set $T$. More precisely, in our construction we associate, for each fixed $\psi_{J}(\Gamma)$ with $J \in \Sigma_{q}^{k}$ (hence a fixed $c_{J}$ ), a set

$$
\Delta(J)=\left\{d_{I}-c_{J}: I \in \Sigma_{N}^{k}, \psi_{J}(\Gamma) \cap \varphi_{I}(\Gamma) \neq \emptyset\right\},
$$

where $\varphi_{I}(x)=A^{-k}\left(x+d_{I}\right)$. We regard $\Delta(J)$ as a symbol attached to $J \in \Sigma_{q}$. Then $\mathcal{S}_{k}=\left\{J \in \Sigma_{q}^{k}: \Delta(J) \neq \emptyset\right\}$. We define

$$
\mathcal{S}_{k}^{o}=\left\{J \in \mathcal{S}_{k}: \Delta(J L) \neq \emptyset, \forall L \in \Sigma_{q}\right\} \quad \text { and } \quad \mathcal{S}_{k}^{\partial}=\mathcal{S}_{k} \backslash \mathcal{S}_{k}^{o} .
$$

These three families of indices will be used to determine $T, T^{\circ}$, and $\partial T$ as in the following theorem:

Theorem 1.2. Let $A$ be an expanding matrix in $M_{n}(\mathbb{Z})$ and let $\mathcal{D} \subseteq \mathbb{Z}^{n}$ be a digit set. With the above notations, we have:

(i) $T=\bigcap_{k=1}^{\infty}\left(\bigcup_{J \in \mathcal{S}_{k}} \psi_{J}(\Gamma)\right)$.

(ii) $T^{o} \neq \emptyset$ if and only if $\mathcal{S}_{k}^{o} \neq \emptyset$, for some $k$ bounded by a constant, depends only on the diameter of $\Gamma$.

(iii) $\partial T=\bigcap_{k=1}^{\infty}\left(\bigcup_{J \in \mathcal{S}_{k}^{\jmath}} \psi_{J}(\Gamma)\right)$. 
The IFS $\left\{\psi_{j}\right\}_{j=1}^{q}$ induces a recurrent relationship on the $\Delta(J)$ 's, which is used to construct the graph-directed system $(V, E)$. Here $V$ has $(m+1)$-vertices formed by the distinct nonempty symbols $\Delta(J)$; the set of outgoing "edges" $E=\left\{E_{i j}\right\}_{0 \leq i, j \leq m}$ satisfies $E_{i j} \subseteq \mathcal{C}$ and $E_{i j} \cap E_{i j^{\prime}}=\emptyset$ if $j \neq j^{\prime}$. The associated IFS is defined by

$$
\left\{\varphi_{i j}^{e}(x)=A^{-1}(x+e): e \in E_{i j}, 0 \leq i, j \leq m\right\} .
$$

The attractor of the system is $\left\{F_{0}, F_{1}, \ldots, F_{m}\right\}$ where $F_{i} \subseteq \Gamma$, and

$$
F_{i}=\bigcup_{j=0}^{m} \bigcup_{e \in E_{i j}} \varphi_{i j}^{e}\left(F_{j}\right):=\bigcup_{j=0}^{m} A^{-1}\left(F_{j}+E_{i j}\right), \quad i=0,1, \ldots, m,
$$

with $F_{0}=T$. If $T^{o} \neq \emptyset$, we consider $\partial T$ by using the $J \in \bigcup_{k} \mathcal{S}_{k}^{\partial}$ to construct a subgraph-directed IFS of (1.3) and has $F_{0}=\partial T$. Let $B=\left(b_{i j}\right)$ be the adjacency matrix defined by $b_{i j}=\# E_{i j}$ for all $0 \leq i, j \leq m$, then we can make use of a theorem of Mauldin and Williams [24] to conclude the following dimension formula (Theorems 4.2 and 4.3).

Theorem 1.3. Let $A=\rho^{-1} R \in M_{n}(\mathbb{Z})$ where $R$ is an orthonormal matrix and $0<$ $\rho<1$, let $\mathcal{D} \subseteq \mathbb{Z}^{n}$ be an integral digit set. Let $B\left(B^{\prime}\right)$ be the adjacency matrix of the graph-directed relationship of $T$ ( $\partial T$, respectively). Then

$$
\operatorname{dim}_{H}(T)=\frac{\log \lambda_{B}}{-\log \rho}, \quad \operatorname{dim}_{H}(\partial T)=\frac{\log \lambda_{B^{\prime}}}{-\log \rho},
$$

where $\lambda_{B}, \lambda_{B^{\prime}}$ are the spectral radius of $B$ and $B^{\prime}$, respectively.

We remark that there are various algorithms to calculate the dimension of the selfsimilar sets. They can be classified into two types: one type is to calculate the Hausdorff dimension of such sets if they have Lebesgue measure zero (e.g., Falconer [8], [9], Rao and Wen [28], Ngai and Wang [26], and Zerner [33]); another type is to calculate the Hausdorff dimension of the boundaries of the self-similar tiles (Strichartz and Wang [30], Duvall et al. [7], and Veerman [31]). Our theorems and the algorithm allow us to have a unified approach to apply to all the cases of integral self-affine sets. Our approach may or may not be as efficient as the other more specialized methods to deal with integral self-affine tiles or self-affine sets with no interior.

For the dimension of self-affine sets, the above theorem is not true in general. McMullen [25] and Bedford [2] had considered the special case for $A=\left(\begin{array}{ll}n & 0 \\ 0 & m\end{array}\right)$ and the IFS has no overlap. Based on the directed graph version of Kenyon and Peres [17] we can use the above construction to include the case that the IFS has overlaps (Theorem 4.4).

It is known that the Lebesgue measure of a self-affine set $T(A, \mathcal{D})$ is an integer when $\# \mathcal{D}=|\operatorname{det}(A)|[19]$. A simple example shows that this is not true if $\# \mathcal{D}>|\operatorname{det}(A)|$ (see Section 4). For the next application of the above construction we prove (Theorem 4.5).

Theorem 1.4. Let $A \in M_{n}(\mathbb{Z})$ be an expanding matrix and let $\mathcal{D} \subseteq \mathbb{Z}^{n}$ be a digit set. Then the Lebesgue measure of the attractor $T$ is a rational number. 
For a self-affine tile it is not so simple to determine its interior (even for the case of the twin dragon). Our other application of the graph-directed system is to give a constructive way to find the interior points (Theorem 4.6).

For the organization of the paper, we introduce the auxiliary system and the symbols $\Delta(J)$ in Section 2 and prove Theorem 1.1. We construct the graph-directed system in Section 3, and for the applications we prove Theorems 1.3 and 1.4 and the interior point question in Section 4. In Section 5, we lay out the algorithm to calculate the dimensions and give some examples to explain the construction of the directed graphs. There are several technicalities in the actual implementation, for these we will make some further discussions and remarks in Section 6.

\section{The Setup}

We let $A \in M_{n}(\mathbb{Z})$ be an expanding integral matrix, $q=|\operatorname{det}(A)|$, and let $\mathcal{D}=$ $\left\{d_{1}, \ldots, d_{N}\right\} \in \mathbb{Z}^{n}$ with $d_{1}=0$. The associated IFS is

$$
\varphi_{i}(x)=A^{-1}\left(x+d_{i}\right), \quad i=1, \ldots, N,
$$

and the self-affine set is $T=T(A, \mathcal{D})$ as described in the Introduction. For the multiindices $I$, we use $|I|$ to denote its length, and let $\Sigma_{N}^{k}:=\left\{I=\left(i_{1}, i_{2}, \ldots, i_{k}\right): i_{j}=\right.$ $1, \ldots, N\} ; \Sigma_{N}:=\bigcup_{k=1}^{\infty} \Sigma_{N}^{k}$. For $I=\left(i_{1}, \ldots, i_{k}\right) \in \Sigma_{N}^{k}$, we write

$\varphi_{I}(x)=\varphi_{i_{1}} \circ \varphi_{i_{2}} \circ \cdots \circ \varphi_{i_{k}}, \quad d_{I}=d_{i_{k}}+A d_{i_{k-1}}+\cdots+A^{k-1} d_{i_{1}}, \quad T_{I}=\varphi_{I}(T)$.

It follows that

$$
\varphi_{I}(x)=A^{-k}\left(x+d_{I}\right) \quad \text { and } \quad T_{I}=A^{-k}\left(T+d_{I}\right),
$$

and for each $k$

$$
T=\bigcup_{|I|=k} \varphi_{I}(T)=A^{-k}\left(T+\mathcal{D}_{k}\right)
$$

where $\mathcal{D}_{k}=\mathcal{D}+A \mathcal{D}+\cdots+A^{k-1} \mathcal{D}$.

Note that, in the above expression, the sets $\left\{\varphi_{I}(T):|I|=k\right\}$ have overlaps in general. Our basic technique is to introduce an auxiliary system to avoid this main difficulty:

(A1) For the above matrix $A$ with $q=|\operatorname{det}(A)|$, we choose a $q$-digit set $\mathcal{C}=$ $\left\{c_{1}, \ldots, c_{q}\right\} \subseteq \mathbb{Z}^{n}$ such that the IFS

$$
\psi_{j}(x)=A^{-1}\left(x+c_{j}\right), \quad j=1, \ldots, q,
$$

generates a self-affine tile $\Gamma$ with $T \subset \Gamma^{o}$ and $\Gamma+\mathcal{D} \subseteq A \Gamma$.

For simple cases, it is quite easy to construct such $\mathcal{C}$ and $\Gamma$. In general, there are some technicalities to determine these auxiliary set constructively. We will discuss this in Section 6. Also the auxiliary tiles and the IFS can be adjusted to serve a different purpose, some modifications of (A1) are at the end of this section. It follows from (A1) that

$$
T=\bigcap_{k=1}^{\infty} \bigcup_{I \in \Sigma_{N}^{k}} \varphi_{I}(\Gamma) \quad \text { and } \quad \Gamma=\bigcup_{J \in \Sigma_{q}^{k}} \psi_{J}(\Gamma) \quad \forall k
$$


For the auxiliary IFS $\left\{\psi_{j}\right\}_{j=1}^{q}$ we have, similar to the previous notations, for $J \in \Sigma_{q}^{k}$,

$$
\psi_{J}(x)=A^{-k}\left(x+c_{J}\right) \quad \text { and } \quad \Gamma_{J}=\psi_{J}(\Gamma)=A^{-k}\left(\Gamma+c_{J}\right),
$$

where $c_{J} \in \mathcal{C}_{k}:=\mathcal{C}+A \mathcal{C}+\cdots+A^{k-1} \mathcal{C}$. For simplicity we also let

$$
\Phi^{(k)}(\Gamma)=\bigcup_{I \in \Sigma_{N}^{k}} \varphi_{I}(\Gamma) \quad \text { and } \quad \Psi^{(k)}(\Gamma)=\bigcup_{J \in \Sigma_{q}^{k}} \psi_{J}(\Gamma) .
$$

Then $T=\bigcap_{k=1}^{\infty} \Phi^{(k)}(\Gamma)$ and $\Gamma=\Psi^{(k)}(\Gamma)$ for each $k$.

For each fixed $\psi_{J}(\Gamma)$ (hence a fixed $\left.c_{J}\right), J \in \Sigma_{q}^{k}, k \geq 1$, we define

$$
\Delta(J)=\left\{d_{I}-c_{J}: I \in \Sigma_{N}^{k}, \psi_{J}(\Gamma) \cap \varphi_{I}(\Gamma) \neq \emptyset\right\} .
$$

In the next section we will use these nonempty $\Delta(J), J \in \Sigma_{q}$, to form a set of vertices of a graph-directed system. First we observe a few direct conclusions from the definition.

Proposition 2.1. By using the above notations, we have:

(i) For $|J|=|I|, \psi_{J}(\Gamma) \cap \varphi_{I}(\Gamma) \neq \emptyset$ if and only if $\left(\Gamma+c_{J}\right) \cap\left(\Gamma+d_{I}\right) \neq \emptyset$.

(ii) If $\Delta(J)=\emptyset$, then $\psi_{J}(\Gamma) \cap T=\emptyset$.

(iii) $\left\{\Delta(J): J \in \Sigma_{q}\right\}$ is a finite family of sets.

Proof. Part (i) follows from

$$
\psi_{J}(\Gamma) \cap \varphi_{I}(\Gamma)=A^{-k}\left(\Gamma+c_{J}\right) \cap A^{-k}\left(\Gamma+d_{I}\right) .
$$

Part (ii) is trivial since $T \subseteq \Phi^{(k)}(\Gamma)$.

For (iii) we observe that $\left(\Gamma+c_{J}\right) \cap\left(\Gamma+d_{I}\right) \neq \emptyset$ implies that $\left\|c_{J}-d_{I}\right\| \leq 2|\Gamma|$. It follows that there are finitely many $c_{J}-d_{I} \in \mathbb{Z}^{n}$ and hence there are at most finitely many $\Delta(J)$.

Let

$$
\begin{aligned}
& \mathcal{S}_{k}=\left\{J \in \Sigma_{q}^{k}: \Delta(J) \neq \emptyset\right\}, \\
& \mathcal{S}_{k}^{o}=\left\{J \in \mathcal{S}_{k}: \Delta(J L) \neq \emptyset, \forall L \in \Sigma_{q}\right\}, \\
& \mathcal{S}_{k}^{\partial}=\mathcal{S}_{k} \backslash \mathcal{S}_{k}^{o},
\end{aligned}
$$

and let

$$
\mathcal{S}=\bigcup_{k=1}^{\infty} \mathcal{S}_{k}, \quad \mathcal{S}^{o}=\bigcup_{k=1}^{\infty} \mathcal{S}_{k}^{o}, \quad \mathcal{S}^{\partial}=\bigcup_{k=1}^{\infty} \mathcal{S}_{k}^{\partial}
$$

We want to use these indices and the auxiliary system $\left\{\psi_{j}\right\}_{j=1}^{q}$ to express $T$. 
Theorem 2.2. Let $A$ be an expanding matrix in $M_{n}(\mathbb{Z})$, let $\mathcal{D} \subseteq \mathbb{Z}^{n}$ be a digit set, and let $T=T(A, \mathcal{D})$, then:

(i) $T=\bigcap_{k=1}^{\infty}\left(\bigcup_{J \in \mathcal{S}_{k}} \psi_{J}(\Gamma)\right)$.

(ii) $T^{o} \neq \emptyset$ if and only if $\mathcal{S}_{k}^{o} \neq \emptyset$ for some $k>0$.

(iii) $\partial T=\bigcap_{k=1}^{\infty}\left(\bigcup_{J \in \mathcal{S}_{k}^{\partial}} \psi_{J}(\Gamma)\right)$.

In particular, if $\mathcal{S}^{\circ}=\emptyset$, then $\mathcal{S}=\mathcal{S}^{\partial}$ and (i) and (iii) coincide.

Remark. The $k$ in (ii) is actually bounded by $2^{\ell}$ where $\ell$ is the total number of integral points contained in the ball $B_{|\Gamma|}$. The explanation is given in Section 5 after Step 3.

Proof. (i) Write $\Gamma=\Psi^{(k)}(\Gamma)=E_{k} \cup \tilde{E}_{k}$ where

$$
E_{k}=\bigcup_{J \in \mathcal{S}_{k}} \psi_{J}(\Gamma) \quad \text { and } \quad \tilde{E}_{k}=\bigcup_{J \in \Sigma_{q}^{k} \backslash \mathcal{S}_{k}} \psi_{J}(\Gamma) .
$$

Then $T \cap \tilde{E}_{k} \subseteq \Phi^{(k)}(\Gamma) \cap \tilde{E}_{k}=\emptyset$. Hence, for each $k$,

$$
T=T \cap \Gamma \subseteq T \cap\left(E_{k} \cup \tilde{E}_{k}\right) \subseteq E_{k} .
$$

Consequently, $T \subseteq \bigcap_{k=1}^{\infty} E_{k}=\bigcap_{k=1}^{\infty} \bigcup_{J \in \mathcal{S}_{k}} \psi_{J}(\Gamma)$.

To prove the reverse inclusion, we let $\varepsilon>0$, then for $k$ large enough, such that $\left|A^{-k} \Gamma\right| \leq \varepsilon / 2$ and $\Phi^{(k)}(\Gamma) \subseteq[T]_{\varepsilon / 2}\left([A]_{\varepsilon}\right.$ denotes the open $\varepsilon$-neighborhood of $\left.A\right)$, the definition of $\mathcal{S}_{k}$ implies that $\psi_{J}(\Gamma) \cap \Phi^{(k)}(\Gamma) \neq \emptyset$ for $J \in \mathcal{S}_{k}$. Hence

$$
\bigcap_{k=1}^{\infty} \bigcup_{J \in \mathcal{S}_{k}} \psi_{J}(\Gamma) \subseteq[T]_{\varepsilon} .
$$

and (i) follows by letting $\varepsilon$ tend to 0 .

(ii) Let $J \in \mathcal{S}_{k}^{o}$, then $J J^{\prime} \in \mathcal{S}_{k+l}^{o}$ for any $J^{\prime} \in \Sigma_{q}^{l}$. Hence

$$
\begin{aligned}
\psi_{J}(\Gamma) & =\bigcap_{l=1}^{\infty} \bigcup_{\left|J^{\prime}\right|=l} \psi_{J J^{\prime}}(\Gamma)=\bigcap_{l=1}^{\infty} \bigcup\left\{\psi_{J J^{\prime}}(\Gamma): J J^{\prime} \in \mathcal{S}_{k+l}^{o}\right\} \\
& \subseteq \bigcap_{m=1}^{\infty} \bigcup\left\{\psi_{J^{\prime}}(\Gamma): J^{\prime} \in \mathcal{S}_{m}\right\}=T \quad \text { (by (i)). }
\end{aligned}
$$

This implies that $T^{o} \neq \emptyset$. Conversely, if $T^{o} \neq \emptyset$, then by (i) there exists $J \in \Sigma_{q}^{k}$ such that $\psi_{J}(\Gamma) \subseteq T^{o}$. Hence $\psi_{J J^{\prime}}(\Gamma) \subseteq T^{o}$ for all $J^{\prime} \in \Sigma_{q}$. This implies that $J \in \mathcal{S}_{k}^{o}$.

(iii) From (i) we have $T \subseteq \bigcup_{J \in \mathcal{S}_{k}} \psi_{J}(\Gamma)$. For any $x \in \partial T$ and $k \geq 1$, we show that $x \in \bigcup_{J \in \mathcal{S}_{k}^{\partial}} \psi_{J}(\Gamma)$. If otherwise, then $x \in \bigcup_{J \in \mathcal{S}_{k}^{o}} \psi_{J}(\Gamma)$. Let

$$
\mathcal{J}_{x}=\left\{J \in \Sigma_{q}^{k}: x \in \psi_{J}(\Gamma) \text { and } J \in \mathcal{S}_{k}^{o}\right\}
$$

and let

$$
F_{k}=\bigcup_{J \in \mathcal{J}_{x}} \psi_{J}(\Gamma), \quad \tilde{F}_{k}=\bigcup_{J \in \Sigma_{q}^{k} \backslash \mathcal{J}_{x}} \psi_{J}(\Gamma) .
$$


Then $x \in F_{k} \backslash \tilde{F}_{k}, \Gamma=F_{k} \cup \tilde{F}_{k}$, and $F_{k} \subseteq T$ (by (2.2)). Since $x \in \partial T \subset \Gamma^{o}$ and $\tilde{F}_{k}$ is compact, there exists an open ball $B_{\delta}(x) \subset \Gamma^{o} \backslash \tilde{F}_{k}$. This implies $B_{\delta}(x) \subseteq F_{k} \subseteq T$ and contradicts that $x \in \partial T$. All the above imply that

$$
\partial T \subseteq \bigcap_{k=1}^{\infty} \bigcup_{J \in \mathcal{S}_{k}^{\partial}} \psi_{J}(\Gamma) .
$$

Conversely, we observe that for $J \in \mathcal{S}_{k}^{\partial}$, either $\psi_{J}(\Gamma) \cap T=\emptyset$ or $\psi_{J}(\Gamma) \cap \partial T \neq \emptyset$. Let $\tilde{\mathcal{S}}_{k}^{\partial}$ be the set of all $J \in \mathcal{S}_{k}^{\partial}$ that satisfies $\psi_{J}(\Gamma) \cap \partial T \neq \emptyset$. Let $\alpha_{k}=\left|A^{-k}(\Gamma)\right|$, then

$$
\bigcup_{J \in \tilde{\mathcal{S}}_{k}^{\partial}} \psi_{J}(\Gamma) \subseteq[\partial T]_{\alpha_{k}}
$$

For any $J^{\prime} \in \mathcal{S}_{k}^{\partial} \backslash \tilde{\mathcal{S}}_{k}^{\partial}$, the definition implies that if $\psi_{J^{\prime}}(\Gamma) \cap T=\emptyset$, then there exists an integer $s$ (depends on $J^{\prime}$ ) such that

$$
\psi_{J^{\prime} J_{1}}(\Gamma) \cap \Phi^{(k+s)}(\Gamma)=\emptyset \quad \forall J_{1} \in \Sigma_{q}^{s},
$$

hence $J^{\prime} J_{1} \notin \mathcal{S}_{k+s}$. Let $s_{k}$ be the maximum among all the $s$ associated with $J^{\prime} \in \mathcal{S}_{k}^{\partial} \backslash \tilde{\mathcal{S}}_{k}^{\partial}$ as above. It follows that for $J \in \mathcal{S}_{k+s_{k}}$ we can write $J=\tilde{J} J_{1}$ with $\tilde{J} \in \tilde{\mathcal{S}}_{k}^{\partial}$ and $J_{1} \in \Sigma_{q}^{s_{k}}$. Therefore

$$
\bigcup_{J \in \mathcal{S}_{k+s_{k}}^{\partial}} \psi_{J}(\Gamma) \subseteq \bigcup_{\tilde{J} \in \tilde{\mathcal{S}}_{k}^{\partial}, J_{1} \in \Sigma_{q}^{s_{k}}} \psi_{\tilde{J} J_{1}}(\Gamma) \subseteq[\partial T]_{\alpha_{k}} .
$$

Consequently, $\bigcap_{i=1}^{\infty} \bigcup_{J \in \mathcal{S}_{i}^{\partial}} \psi_{J}(\Gamma) \subseteq[\partial T]_{\alpha_{k}}$ and (iii) follows by letting $k$ tend to $\infty$.

For the auxiliary tile we assume in (A1) that $T \subset \Gamma^{o}$. In many practical cases, it is more convenient to assume that $T \subseteq \Gamma$ instead. For example, in the consideration of the Cantor set in $[0,1]$ in Example 5.1 (and the other examples in that section), it will be simpler to use the auxiliary tile $\Gamma=[0,1]$ instead of using $[-1,2]$ in order to satisfy $T \subset \Gamma^{o}$. We therefore readjust the auxiliary system (A1) as:

$\left(\mathrm{A} 1^{\prime}\right)$ Let $\mathcal{C}=\left\{c_{1}, \ldots, c_{q}\right\} \subseteq \mathbb{Z}^{n}$ such that the IFS

$$
\psi_{j}(x)=A^{-1}\left(x+c_{j}\right), \quad j=1, \ldots, q,
$$

generates a self-affine tile $\Gamma$ with $T \subseteq \Gamma$ and $\Gamma+\mathcal{D} \subseteq A \Gamma$.

We define the symbols as

$$
\Delta^{\prime}(J)=\left\{d_{I}-c_{J}: I \in \Sigma_{N}^{k}, \psi_{J}\left(\Gamma^{o}\right) \cap \varphi_{I}\left(\Gamma^{o}\right) \neq \emptyset\right\}
$$

and replace the group of notations for $\mathcal{S}$ by $\mathcal{S}^{\prime}$. There is basically no change of Proposition 2.1 and Theorem 2.2 (except (iii)). 
Proposition 2.1'. With the modified $\left(A 1^{\prime}\right)$, we have:

(i) For $|J|=|I|, \psi_{J}\left(\Gamma^{o}\right) \cap \varphi_{I}\left(\Gamma^{o}\right) \neq \emptyset$ if and only if $\left(\Gamma^{o}+c_{J}\right) \cap\left(\Gamma^{o}+d_{I}\right) \neq \emptyset$.

(ii) If $\Delta^{\prime}(J)=\emptyset$, then $\psi_{J}(\Gamma) \cap T^{o}=\emptyset$.

(iii) $\left\{\Delta^{\prime}(J): J \in \Sigma_{q}\right\}$ is a finite family of sets.

Theorem 2.2'. With the modified $\left(A 1^{\prime}\right)$, we have:

(i) $T=\bigcap_{k=1}^{\infty}\left(\bigcup_{J \in \mathcal{S}_{k}^{\prime}} \psi_{J}(\Gamma)\right)$.

(ii) $T^{o} \neq \emptyset$ if and only if $\mathcal{S}_{k}^{\prime o} \neq \emptyset$ for some $k>0$.

(iii) Let $G=\bigcap_{k=1}^{\infty}\left(\bigcup_{J \in \mathcal{S}_{k}^{\prime}} \psi_{J}(\Gamma)\right)$, then $G \subseteq \partial T \subseteq \partial \Gamma \cup G$.

Proof. For (i) we define, in the same way, $E_{k}=\bigcup_{J \in \mathcal{S}_{k}^{\prime}} \psi_{J}(\Gamma)$ and $\tilde{E}_{k}=\bigcup_{J \in \Sigma_{q}^{k} \mathcal{S}_{k}^{\prime}}$ $\psi_{J}(\Gamma)$. By replacing $T$ with $T^{o}$ in the proof of Theorem 2.2(i), we have $T^{o} \subseteq E_{k}$. This implies that $T \subseteq E_{k}=\bigcup_{J \in \mathcal{S}_{k}} \psi_{J}(\Gamma)$ for all $k$. The reverse inclusion can be proved in the same way.

The proof of (ii) has no change.

For (iii), there is no change for $G \subseteq \partial T$. For the other inclusion, we let $x \in \partial T \backslash \partial \Gamma$ and show that $x \in \bigcup_{J \in \mathcal{S}_{k}^{\prime}} \psi_{J}(\Gamma)$. Assume the contrary, then the same setup with $x \notin \partial \Gamma$ will imply that $x \in \Gamma^{o}$. We can then use the same proof in Theorem 2.2(iii).

Remark 2.3. We can also define

$$
\Delta^{\prime}(J)=\left\{d_{I}-c_{J}: I \in \Sigma_{N}^{k}, \psi_{J}(\Gamma) \cap \varphi_{I}(\Gamma) \neq \emptyset\right\} .
$$

Then Theorem $2.2^{\prime}$ holds in the same way. We will use this setup in Theorem 4.5.

Remark 2.4. The set-back to use (A1') instead of (A1) in Theorem 2.2'(iii) cannot be improved to the form in Theorem 2.2(iii). Indeed if we consider the trivial case: $A=2, \mathcal{D}=\{0,1\}$ on $\mathbb{R}$, then $T=[0,1]$. Let $\mathcal{C}=\{0,1\}$, then $\Gamma=[0,1]$ is the auxiliary tile. It is easy to show that $\mathcal{S}^{\prime}=\mathcal{S}^{\prime o}$, so that $\mathcal{S}^{\prime 2}=\emptyset$.

Remark 2.5. Also in Theorem 2.2'(iii), if $\operatorname{dim}_{H} \partial T>\operatorname{dim}_{H} \partial \Gamma$, then $\operatorname{dim}_{H} \partial T=$ $\operatorname{dim}_{H} G$. The latter term can be calculated by a graph-directed system in the same way as Theorem 2.2 and will be discussed in the next section.

\section{The Graph-Directed System}

We first prove a recurrent relationship on the set of $\Delta(\Gamma)$ 's.

Proposition 3.1. For any $J, J^{\prime} \in \Sigma_{q}$, if $\Delta(J)=\Delta\left(J^{\prime}\right)$, then $\Delta(J j)=\Delta\left(J^{\prime} j\right)$ for $j=1, \ldots, q$.

Proof. It suffices to show that $\Delta(J j) \subseteq \Delta\left(J^{\prime} j\right)$; the reverse inclusion will hold in the same way. Let $|J|=k$ and let $\alpha \in \Delta(J j)$ then, by definition, $\alpha=\delta-c_{J j}$ for some 
$\delta \in \mathcal{D}_{k+1}$ and

$$
\left(\Gamma+c_{J j}\right) \cap(\Gamma+\delta) \neq \emptyset .
$$

Note that $c_{J j}=A c_{J}+c_{j}$ and $\delta=A d_{I}+d_{i}$ for some $d_{I} \in \mathcal{D}_{k}, d_{i} \in \mathcal{D}$, we have from (3.1) and $\Gamma+\mathcal{D} \subseteq A \Gamma$ that

$$
\left(\Gamma+c_{J}\right) \cap\left(\Gamma+d_{I}\right) \supseteq A^{-1}\left(\Gamma+c_{J j}\right) \cap A^{-1}(\Gamma+\delta) \neq \emptyset .
$$

This implies that $d_{I}-c_{J} \in \Delta(J)$. Now consider the hypothesis $\Delta(J)=\Delta\left(J^{\prime}\right)$, let $|J|=k,\left|J^{\prime}\right|=l$, then

$$
d_{I}-c_{J}=d_{I^{\prime}}-c_{J^{\prime}}
$$

for some $d_{I^{\prime}} \in \mathcal{D}_{l}$. By adding and subtracting, we have, from (3.1),

$$
\begin{aligned}
\emptyset & \neq\left(\Gamma+A c_{J^{\prime}}+c_{j}\right) \cap\left(\Gamma+\delta+A c_{J^{\prime}}-A c_{J}\right) \\
& =\left(\Gamma+c_{J^{\prime} j}\right) \cap\left(\Gamma+\delta+A d_{I^{\prime}}-A d_{I}\right) \\
& =\left(\Gamma+c_{J^{\prime} j}\right) \cap\left(\Gamma+A d_{I^{\prime}}+d_{i}\right) \\
& =\left(\Gamma+c_{J^{\prime} j}\right) \cap\left(\Gamma+\delta^{\prime}\right),
\end{aligned}
$$

where $\delta^{\prime}=A d_{I^{\prime}}+d_{i} \in \mathcal{D}_{l+1}$. This shows that

$$
\alpha=\delta-c_{J j}=\delta^{\prime}-c_{J^{\prime} j} \in \Delta\left(J^{\prime} j\right)
$$

and the claim $\Delta(J j) \subseteq \Delta\left(J^{\prime} j\right)$ follows.

For convenience, we add a new element $J=0$ to the index set $1, \ldots, q$, let $\Sigma_{q}^{*}=$ $\left\{0 J: J \in \Sigma_{q}\right\} \cup\{0\}$, and define $\Delta(0)=\{0\}$ and $\Delta(0 J)=\Delta(J)$. We construct a finite graph-directed set $(V, E)$ as follows: we start with the symbol $\{0\}$, by using the IFS with the seed set $\Gamma$, the descendants are $\psi_{i}(\Gamma), i=1, \ldots, q$. We define the corresponding iteration on the symbols as

$$
\Delta(0) \rightarrow\{\Delta(1), \Delta(2), \ldots, \Delta(q)\} .
$$

In general, for $\psi_{J}(\Gamma)$, the descendents are $\left\{\psi_{J i}(\Gamma)\right\}_{i=1}^{q}$ and the actions on the symbols are

$$
\Delta(J) \rightarrow\{\Delta(J 1), \Delta(J 2), \ldots, \Delta(J q)\} .
$$

By the proof of Proposition 2.1(iii), the set $V$ of nonempty $\Delta(J), J \in \Sigma_{q}$, is a finite set and can be determined within a fixed step. We will relabel the indices of the $J$ 's in $\Delta(J)$ to form a set of vertices

$$
V=\left\{\Delta(0)=\{0\}, \Delta\left(J_{1}\right), \ldots, \Delta\left(J_{m}\right)\right\}
$$

For brevity we write $V=\left\{v_{0}, \ldots, v_{m}\right\}$. Next we define the directed edges $E=\left\{E_{i j}\right\}_{i, j=0}^{m}$ on $V$ by

$$
E_{i j}=\left\{c_{s}: \Delta\left(J_{i} s\right)=\Delta\left(J_{j}\right), 1 \leq s \leq q\right\}
$$


(and $=\emptyset$ if $\Delta\left(J_{i} s\right) \neq \Delta\left(J_{j}\right)$ for all $\left.1 \leq s \leq q\right)$. The definition is well-defined by Proposition 3.1. It is clear that $E_{i j} \subseteq \mathcal{C} ; E_{i j} \cap E_{i j^{\prime}}=\emptyset$ if $j \neq j^{\prime}$. Note that it is possible that, for some $v_{i}$, the corresponding $E_{i, j}$ are empty for all $j$. We will discard those $v_{i}$ 's where there is no path going out, then discard all the paths going to such discarded vertices. Hence, without loss of generality, we assume that, for each $i$,

$$
1 \leq \sum_{j=0}^{m} \# E_{i j} \leq q
$$

Remark 3.2. The graph-directed set $(V, E)$ can also be viewed as follows: let $\mathcal{S}^{*}=$ $\{0 J: J \in \mathcal{S}\} \cup\{0\}$ by joining the index 0 to $\mathcal{S}$. We use $\Delta$ to define an equivalent relationship on $\mathcal{S}$ and denote the quotient by $\mathcal{S}^{*} / \Delta$. It is easy to see that the equivalent classes are in one-to-one correspondence with the vertices in $V$ (after discarding those vertices with no path going out). The iteration in (3.2) induces the map

$$
\mathcal{S}_{k}^{*} / \Delta \rightarrow \mathcal{S}_{k+1}^{*} / \Delta, \quad[J] \rightarrow\{[J j]\}_{j=1}^{q} .
$$

This is well-defined in view of Proposition 3.1. The corresponding directed edges from $[J]$ to $[J j]$ are just the set of $j^{\prime \prime}$ s such that $[J j]=\left[J^{\prime}\right]$.

By using the set $(V, E)$, we define the graph-directed IFS as

$$
\varphi_{i j}^{e}(x)=A^{-1}(x+e) \quad \text { for } \quad e \in E_{i j} \neq \emptyset, \quad i, j=0,1, \ldots, m .
$$

Proposition 3.3. For the above graph-directed IFS, there exist compact subsets $\left\{F_{0}\right.$, $\left.F_{1}, \ldots, F_{m}\right\}$ such that

$$
F_{i}=\bigcup_{j=0}^{m} \bigcup_{e \in E_{i j}} \varphi_{i j}^{e}\left(F_{j}\right)=\bigcup_{j=0}^{m} A^{-1}\left(F_{j}+E_{i j}\right), \quad i=0,1, \ldots, m,
$$

where $E_{i j} \subseteq \mathcal{C}, E_{i j} \cap E_{i j^{\prime}}=\emptyset$ if $j \neq j^{\prime}$. Moreover, $F_{0}=T$ and for each $i$, and the $F_{j}+E_{i j}$ in the union are measurably disjoint.

Proof. The existence of the invariant sets in (3.6) is well-known [9, p. 48], [24]. Note that, for each $i$,

$$
F_{i}=\left\{\sum_{k=1}^{\infty} A^{-k} e_{k}: e_{k} \in E_{i_{k} i_{k+1}} \neq \emptyset \text { for } i_{1}=i, 0 \leq i_{k} \leq m, k \in \mathbb{N}\right\}
$$

(see [11]) and $F_{i} \subseteq \Gamma$ because $E_{i j} \subseteq \mathcal{C}$. Since $\Gamma+c, c \in \mathcal{C}$, are measurably disjoint and $E_{i j} \cap E_{i j^{\prime}}=\emptyset$ if $j \neq j^{\prime}$, they imply that for each $i, \bigcup_{j=0}^{m}\left(F_{j}+E_{i j}\right)$ is a measurably disjoint union. To see that $F_{0}=T$, we observe that

$$
\left\{\sum_{s=1}^{k} A^{-s} e_{s}: e_{s} \in E_{i_{s}, i_{s+1}} \neq \emptyset \text { for } i_{1}=0,0 \leq i_{s} \leq m\right\}=\left\{A^{-k} c_{J}: J \in \mathcal{S}_{k}\right\}
$$

which converges to $F_{0}$ as $k \rightarrow \infty$. Hence

$$
T=\lim _{k \rightarrow \infty} \bigcup_{J \in \mathcal{S}_{k}} \psi_{J}(\Gamma)=\lim _{k \rightarrow \infty} \bigcup_{J \in \mathcal{S}_{k}} A^{-k}\left(\Gamma+c_{J}\right)=F_{0} .
$$


In the above construction, we use all the nontrivial symbols $\Delta(J)$ to form the set of vertices $V$. For the case $\mathcal{S}^{o} \neq \emptyset$, we can just use the indices $J^{\prime} \in \mathcal{S}^{\partial}$ and the corresponding $\Delta(J)$ to construct a new set of vertices $V^{\partial}=\left\{\Delta(0), \Delta\left(J_{1}^{\prime}\right), \ldots, \Delta\left(J_{k}^{\prime}\right)\right\}$. Analogous to the above we can define edges $E^{\partial}=\left\{E_{i j}\right\}_{0 \leq i, j \leq k}$ and a sub-graph-directed set $\left(V^{\partial}, E^{\partial}\right)$.

Proposition 3.4. With the graph-directed set $\left(V^{\partial}, E^{\partial}\right)$ and the corresponding IFS $\left\{\varphi_{i j}^{e}\right.$ : $\left.e \in E_{i, j}, 0 \leq i, j, \leq k\right\}$ constructed from the index set $\mathcal{S}^{\partial}$, we have $F_{0}=\partial T$ and the other conclusions are the same as in Proposition 3.3.

Remark 3.5. If we use the auxiliary system in $\left(\mathrm{A}^{\prime}\right)$, we can use the symbols $\Delta^{\prime}(J)$ and the same method to define the two graph-directed systems. The systems will be of smaller size.

\section{Some Applications}

Application I. The Hausdorff Dimension of Self-Similar Sets

For the graph-directed relationship (3.6) we can define an $(m+1) \times(m+1)$ adjacency matrix $B$ with the $(i, j)$ th entry given by

$$
b(i, j)=\# E_{i j}, \quad i, j=0,1, \ldots, m,
$$

[9, p. 48]. The adjacency matrix is used to count the paths of the graph-directed set in the iteration. Let $\mathbf{1}$ be the $(m+1)$-vector with all entries equal to 1 and let $\mathbf{e}_{\mathbf{i}}$ be an $(m+1)$-vector with the $i$ th entry 1 and the others zero.

Proposition 4.1. With the above notations, we have

$$
\# \mathcal{S}_{k}=\mathbf{e}_{0}^{t} B^{k} \mathbf{1}
$$

Proof. The entry $b_{k}\left(0, i_{k}\right)$ of $B^{k}$ is given by

$$
b\left(0, i_{k}\right)=\sum_{i_{1}, \ldots, i_{k-1}} b\left(0, i_{1}\right) b\left(i_{1}, i_{2}\right) \cdots b\left(i_{k-1}, i_{k}\right) .
$$

This expression is the number of paths from $v_{0}$ to $v_{i_{k}}$. There are corresponding $J^{*}=$ $0 J=0 j_{1} \cdots j_{k} \in \Sigma_{k}^{*}$ to go from $v_{0}$ to $\Delta\left(0 j_{1} \cdots j_{k}\right)=v_{i_{k}}$. In view of (3.5) and the remark there, the total number of $J$ that satisfies $\Delta(0 J)=v_{i_{k}}$ is given by (4.1). The proposition follows from this since $e_{0}^{t} B^{k} 1$ counts all the paths of length $k$, starting from $v_{0}$.

Theorem 4.2. Let $A=\rho^{-1} R \in M_{n}(\mathbb{Z})$ be an expanding similarity with $0<\rho<1$ and let $\mathcal{D} \subset \mathbb{Z}^{n}$ be a finite digit set. Let $B$ be the adjacency matrix of the graph-directed relationship of $T$. Then

$$
\operatorname{dim}_{H}(T)=\frac{\log \lambda_{B}}{-\log \rho}
$$

where $\lambda_{B}$ is the spectral radius of $B$. 
In view of Proposition 3.3, the theorem is a direct consequence of a theorem of Mauldin and Williams [24]. The basic idea is to apply Proposition 4.1 to obtain

$$
\lambda_{B}=\lim _{k \rightarrow \infty}\left\|B^{k}\right\|^{1 / k}=\lim _{k \rightarrow \infty}\left(\mathbf{e}_{0}^{t} B^{k} \mathbf{1}\right)^{1 / k}=\lim _{k \rightarrow \infty}\left(\# \mathcal{S}_{k}\right)^{1 / k} .
$$

For $\delta_{k}=\left|A^{-k}(\Gamma)\right|=\rho^{k}|\Gamma|,\left\{A^{-k} c_{J}: J \in \mathcal{S}_{k}\right\}$ will give a $\delta_{k}$-ball covering of $T$ because

$$
T \subseteq \bigcup_{J \in \mathcal{S}_{k}} A^{-k}\left(\Gamma+c_{J}\right) \subseteq[T]_{\delta} .
$$

The number in the covering is $\# \mathcal{S}_{k}$ and, in essence, the smallest number needed. From this we see that

$$
\operatorname{dim}_{H}(T)=\operatorname{dim}_{B}(T)=\lim _{k \rightarrow \infty} \frac{\log \# \mathcal{S}_{k}}{-k \log \rho}=\frac{\log \lambda_{B}}{-\log \rho}
$$

If $\mathcal{S}^{o} \neq \emptyset$, then following Proposition 3.5, we can consider $\partial T$ by restricting the indices to $\mathcal{S}^{\partial}$ and set up a subgraph $\left(V^{\partial}, E^{\partial}\right)$ of $(V, E)$. Similar to Theorem 4.2 we have

Theorem 4.3. Let $A=\rho^{-1} R \in M_{n}(\mathbb{Z})$ be an expanding similarity with $0<\rho<1$ and let $\mathcal{D} \subset \mathbb{Z}^{n}$ be a finite digit set. Suppose $T^{o} \neq \emptyset$. Let $B^{\prime}$ be the adjacency matrix corresponding to the subgraph $\left(V^{\partial}, E^{\partial}\right)$. Then

$$
\operatorname{dim}_{H}(\partial T)=\frac{\log \lambda_{B^{\prime}}}{-\log \rho}
$$

where $\lambda_{B^{\prime}}$ is the spectral radius of $B^{\prime}$.

We have the same Theorems 4.2 and 4.3 if we use the auxiliary system in (A1').

\section{Application II. The Hausdorff Dimension of the McMullen-Type Self-Affine Sets}

If $A$ is not a similarity, then the only way we can handle this is for $A=\left(\begin{array}{ll}n & 0 \\ 0 & m\end{array}\right)$, $0<m<n$, the special case of McMullen [25] and Bedford [2]. Let $\mathcal{D} \subseteq \mathbb{Z}_{+}^{2}$ be a digit set. (Note that in [25], [1], $\mathcal{D} \subseteq\{0, \ldots, n-1\} \times\{0, \ldots, m-1\}$ and it is a nonoverlapping case.) Let

$$
\mathcal{C}=\{0, \ldots, \alpha(n-1)\} \times\{0, \ldots, \alpha(m-1)\}:=\left\{c_{j}: j=1,2, \ldots, m n\right\},
$$

where $\alpha=\left[\max _{1 \leq i \leq m n}\left\{d_{i}^{1} /(n-1), d_{i}^{2} /(m-1)\right\}\right]+1$ with $d_{i}=\left(d_{i}^{1}, d_{i}^{2}\right)^{t}$, and let $[x]$ denote the largest integer less than $x$. Let $\Gamma$ be the corresponding rectangular tile, then $T \subseteq \Gamma$ and $\Gamma+\mathcal{D} \subseteq A \Gamma$. By using $\left(\mathrm{A}^{\prime}\right)$ we have a graph-directed system with vertices $V=\left\{v_{0}, \ldots, v_{l}\right\}$ that satisfies (3.6). The system can be constructed with respect to $T$ or to $\partial T$, as in Proposition 3.5. Let $B$ be the adjacency matrix $B=\left(b_{i j}\right)$. Following the setup of Kenyon and Peres [17] we can decompose the $B$ to $m$ matrix $B_{u}$ according to the second coordinate of elements of the edges in $E_{i j}$ where $0 \leq i, j \leq l$ :

$$
b_{i j}^{u}=\#\left\{(w, u): \alpha(w, u) \in E_{i j}\right\}, \quad i, j=0,1, \ldots, l \text { and } u=0,1, \ldots, m-1 .
$$

We can then apply [17, Theorem 2.2] to conclude 
Theorem 4.4. Let $\left\{B_{u}\right\}_{u=0}^{m-1}$ be defined as above. Then

$$
\operatorname{dim}_{H} \tilde{T}=\lim _{k \rightarrow \infty} \frac{1}{k} \log _{m} \sum_{0 \leq i_{1}, \ldots, i_{k} \leq m-1}\left\|B_{i_{k}} B_{i_{k-1}} \cdots B_{i_{1}}\right\|^{\alpha},
$$

where $\alpha=\log m / \log n \leq 1$ and $\tilde{T}=T$ or $\partial T$ according to the directed graphs used.

We remark that the limit in Theorem 4.4 has the same form, with the $\alpha$-norm joint spectral radius, of matrices defined by Jia [15], the difference between them is the range of the values of $\alpha$.

We will illustrate this in Example 5.6. It is also known that for this case the Hausdorff dimension and the box dimension are not the same in general [25]. By using [17, Proposition 3.5], we can also calculate the box dimension of the set $T$. We omit the details of that.

\section{Application III. The Lebesgue Measure of the Self-Similar Regions}

Our next application of the graph-directed IFS is to consider the Lebesgue measure of $T$. Let $\mu$ denote the Lebesgue measure. It is known that for an integral self-affine tile, its Lebesgue measure is an integer [19]. However, this is not true for an integral self-affine region in general, as is shown by the following simple example:

Example. Consider the one-dimensional case, let $A=3, \mathcal{D}=\{0,1,5,6\}$. Then $T=\left[0, \frac{4}{3}\right] \cup\left[\frac{5}{3}, 3\right]$ is the self-affine region, as it satisfies $A T=T+\mathcal{D}$. The Lebesgue measure of $T$ is $\frac{8}{3}$.

Theorem 4.5. Let $A \in M_{n}(\mathbb{Z})$ be an expanding matrix and let $\mathcal{D} \subseteq \mathbb{Z}^{n}$ be a digit set. Then the Lebesgue measure of the self-similar set $T$ is a rational number.

Proof. Without loss of generality, we assume that $\mu(T)>0$, and it follows that $\# \mathcal{D} \geq|\operatorname{det}(A)|$. Let $\left\{F_{0}=T, F_{1}, \ldots, F_{m}\right\}$ be defined as in Proposition 3.3. From (3.6) we have

$$
F_{0}=\bigcup_{j=0}^{m} A^{-1}\left(F_{j}+E_{0 j}\right) .
$$

By taking the Lebesgue measure on both sides of (3.6), and making use of the measurable disjointness of the $F_{j}+E_{i j}$, we have

$$
q \mathbf{u}=B \mathbf{u},
$$

where $q=|\operatorname{det}(A)|, B$ is the adjacency matrix, and $\mathbf{u}=\left(\mu\left(F_{0}\right), \mu\left(F_{1}\right), \ldots, \mu\left(F_{m}\right)\right)^{t}$. We can rearrange the states of $B$ into communication classes and, hence, assume, without loss of generality, that $B$ is of the upper triangular form, i.e.,

$$
B=\left(\begin{array}{llll}
B_{11} & B_{12} & \cdots & B_{1 k} \\
& B_{22} & \cdots & B_{2 k} \\
& & \ddots & \vdots \\
& & & B_{k k}
\end{array}\right) .
$$


Let us write $\mathbf{u}=\left(\mathbf{u}_{1}, \ldots, \mathbf{u}_{k}\right)$ for the corresponding coordinates. Consider the last component

$$
q \mathbf{u}_{k}=B_{k k} \mathbf{u}_{k} .
$$

If $\mathbf{u}_{k}=0$, we have $q \mathbf{u}_{k-1}=B_{(k-1)(k-1)} \mathbf{u}_{k-1}$, and we consider this instead. Hence we can assume that $\mathbf{u}_{k} \neq 0$ and that this is a $q$-eigenvector of $B_{k k}$. This and the fact that the sum of each row of $B_{k k}$ is at most $q$ imply that the row sum must be $q$ [29, Exercise 1.16]. Let $V_{k}$ denote the vertices corresponding to the states in $B_{k k}$, then by (3.6) we have

$$
F_{i}=\bigcup_{j} A^{-1}\left(F_{j}+E_{i j}\right),
$$

where the $i, j$ 's, corresponding to vertices $v_{i}, v_{j} \in V_{k}$, and $\bigcup_{j} E_{i j}=\mathcal{C}$ and $E_{i j} \cap E_{i j^{\prime}}=\emptyset$ if $j \neq j^{\prime}$. By the radix expansion of the $F_{i}$ in $(3.7)$, we see that $F_{i}=\Gamma(=\Gamma(A, \mathcal{C}))$. Since $\Gamma$ is a tile, $\mu(\Gamma)$ is integer-valued [19]. It follows that

$$
\mathbf{u}_{k}=(\mu(\Gamma), \ldots, \mu(\Gamma))
$$

is a vector with integer entries.

Next we consider

$$
q\left(\begin{array}{l}
\mathbf{u}_{k-1} \\
\mathbf{u}_{k}
\end{array}\right)=\left(\begin{array}{ll}
B_{(k-1)(k-1)} & B_{(k-1) k} \\
& B_{k k}
\end{array}\right)\left(\begin{array}{l}
\mathbf{u}_{k-1} \\
\mathbf{u}_{k}
\end{array}\right) .
$$

If $B_{(k-1) k}=0$, then $q \mathbf{u}_{k-1}=B_{(k-1)(k-1)} \mathbf{u}_{k-1}$ and $\mathbf{u}_{k-1}$ is an integral eigenvector as above; if $B_{(k-1) k} \neq 0$, then some of the row-sums of $B_{(k-1)(k-1)}$ are less than $q$. Hence the maximal eigenvalue of $B_{(k-1)(k-1)}$ is less than $q$. It follows that $q E-B_{(k-1)(k-1)}$ is invertible (where $E$ is the identity matrix) and hence

$$
\mathbf{u}_{k-1}=\left(q E-B_{(k-1)(k-1)}\right)^{-1} B_{k k} \mathbf{u}_{k}
$$

is a rational eigenvector. Inductively, we conclude that $\mathbf{u}$ is a rational vector so that $\mu(T)=\mu\left(F_{0}\right)$, the first coordinate of $\mathbf{u}$, is a rational number.

We remark that the proof of Theorem 4.5 actually provides a method of computing the Lebesgue measure of a self-affine set (i.e., the first coordinate of $\mathbf{u}_{1}$ ), if we choose an auxiliary self-affine tile $\Gamma$ whose Lebesgue measure is known (i.e., $\left.\mathbf{u}_{k}=(\mu(\Gamma), \ldots, \mu(\Gamma))\right)$

\section{Application IV. The Interior of a Self-Affine Tile}

For a self-affine tile it is not clear how to determine a point to be in the interior. In this application we will assume that $T=T(A, \mathcal{D})$ itself is a self-affine tile and that we can make use of the present setup to give a constructive way of finding the interior points. In view of $\left(\mathrm{A}^{\prime}\right)$, we take

$$
\begin{aligned}
\mathcal{C}=\mathcal{D}=\left\{d_{0}, \ldots, d_{q-1}\right\} \quad \text { and } \quad \psi_{i}(x)=\varphi_{i}(x) & =A^{-1}\left(x+d_{i}\right), \\
i & =0, \ldots, q-1,
\end{aligned}
$$


themselves to form the auxiliary system, hence $T=\Gamma$. To simplify notation we will omit all the "'"' 's in the set of notations for $\left(\mathrm{A} 1^{\prime}\right)$. For the $\Delta(J)$, we use the definition in Remark 2.3, i.e.,

$$
\Delta(J)=\left\{d_{I}-d_{J}: I \in \Sigma_{N}^{k}, \varphi_{J}(T) \cap \psi_{I}(T) \neq \emptyset\right\} .
$$

In this definition, the $\Delta(J)$ counts the $T_{J^{\prime}}$ in the neighborhood of $T_{J}$. We say that $\Delta(J)$ is maximal if there is no $J^{\prime}$ such that $\Delta(J)$ is a proper subset of $\Delta\left(J^{\prime}\right)$. Intuitively, the $T_{J}$ must be in the interior of $T$. In fact, we have

Theorem 4.6. Suppose $T=T(A, \mathcal{D})$ is a self-affine tile. If $\Delta(J), J \in \Sigma_{q}$, is maximal, then $T_{J} \subseteq T^{o}$.

Proof. We first show that for any $I, J \in \Sigma_{q}, \Delta(J) \subseteq \Delta(I J)$ : let $k=|J|$. For $t \in \Delta(J)$, by definition, there exists $J_{1} \in \Sigma_{q}^{k}$ such that

$$
T_{J_{1}} \cap T_{J} \neq \varnothing \quad \text { and } \quad t=d_{J_{1}}-d_{J} .
$$

It follows that $T_{I J_{1}} \cap T_{I J}=\varphi_{I}\left(T_{J_{1}} \cap T_{J}\right) \neq \emptyset$ and

$$
d_{I J_{1}}-d_{I J}=A^{k} d_{I}+d_{J_{1}}-\left(A^{k} d_{I}+d_{J}\right)=d_{J_{1}}-d_{J}=t .
$$

Hence $t \in \Delta(I J)$ and $\Delta(J) \subseteq \Delta(I J)$.

If $T_{J} \nsubseteq T^{o}$, then there exists an $x \in T_{J} \cap \partial T$. We fix an $I \in \Sigma_{q}$ such that $T_{I} \subset T^{o}$, then $\varphi_{I}(x) \in T_{I} \cap T_{I^{\prime}}$ for some $I^{\prime} \neq I$ and $\left|I^{\prime}\right|=|I|$. We claim that the above $\Delta(J) \subseteq \Delta(I J)$ is a strict inclusion, hence $\Delta(J)$ is not maximal and completes the proof.

To prove the claim we observe that $\varphi_{I}(x) \in T_{I J}$ and $\varphi_{I}(x) \in T_{I^{\prime}}$ imply that there exists an $L^{\prime} \in \Sigma_{q}^{k}$ such that $\varphi_{I}(x) \in T_{I^{\prime} L^{\prime}}$. Then $d_{I^{\prime} L^{\prime}}-d_{I J} \in \Delta(I J)$ by the definition of $\Delta(I J)$. It remains to see that $d_{I^{\prime} L^{\prime}}-d_{I J} \notin \Delta(J)$. For, otherwise, there is an $L \in \Sigma_{q}^{k}$ such that

$$
d_{I^{\prime} L^{\prime}}-d_{I J}=d_{L}-d_{J}=d_{I L}-d_{I J} .
$$

Hence $d_{I^{\prime} L^{\prime}}=d_{I L}$. This contradicts that for a self-affine tile, the elements in $\mathcal{D}_{k}=$ $\mathcal{D}+A \mathcal{D}+\cdots+A^{k-1} \mathcal{D}$ are all distinct [19].

\section{Examples}

We summarize the foregoing sections as an algorithm to find the graph-directed sets $(V, E)$ and $\left(V^{\partial}, E^{\partial}\right)$ : Let $A$ be the expanding integral matrix with $|\operatorname{det}(A)|=q$ and let $\mathcal{D} \subseteq \mathbb{Z}^{n}$ be an $N$-digit set. Let $\left\{\varphi_{i}\right\}_{i=1}^{N}$ be the corresponding IFS and let $T=T(A, \mathcal{D})$ be the self-affine set.

Step 1. We use either (A1) or (A1') to find the auxiliary digit sets $\mathcal{C}$ and $\Gamma$ (the difference is $T \subseteq \Gamma$ versus $T \subseteq \Gamma^{o}$ ). We will discuss some of the technicalities in the next section.

Step 2. Use (3.2), (3.3), and (3.4) to select the nonempty $\Delta(J)$ 's and the set of edges $E$. Discard all those vertices with no edges going out, and then all the edges that go to the removed edges. This way we form the graph-directed set $(V, E)$. The paths in $(V, E)$ are in one-to-one correspondence to the indices in $\mathcal{S}$. 
Step 3. To check that $\mathcal{S}^{o} \neq \emptyset$, we look for those $v \in V$ such that each of its descendents has $q$ edges going out. We then discard all those $v$ and the edges going into them and form a graph-directed subset $\left(V^{\partial}, E^{\partial}\right)$. The corresponding indices are $\mathcal{S}^{\partial}$.

In view of Propositions 2.1(iii) and 3.1, the sets $\Delta(J), J \in \Sigma_{q}$, are integral sets contained in the ball $B_{|\Gamma|}$. Let $l$ be the total number of integral points in the ball, there are at most $2^{l}$ distinct $\Delta(J)$ and within $k \leq 2^{\ell}$ steps, all the $\Delta(J)$ will appear and we can determine $\mathcal{S}_{k}^{o} \neq \emptyset$ in not more than $2^{l}$ steps. This justifies the estimation of $k$ in Theorem 1.2 (or the Remark following Theorem 2.2).

In the following we will give some simple examples to illustrate the algorithm. We use $\left(\mathrm{A} 1^{\prime}\right)$ because the size of the graph-directed system can be reduced significantly. In all the examples we always have $\Gamma+\mathcal{D} \subseteq A \Gamma$. First we wish to test the algorithm on the Cantor set.

Example 5.1. Let $A=3$ and $\mathcal{D}=\{0,2\}$. Then $\operatorname{dim}_{H}(T)=\log 2 / \log 3$.

Solution. We choose $\mathcal{C}=\{0,1,2\}$ and $\Gamma=[0,1]$. It follows from a direct checking of the definition that (using the previous indices: $c_{1}=0, c_{2}=1$, and $c_{3}=2$ )

$$
\Delta(0)=\{0\}, \quad \Delta(1)=\{0\}, \quad \Delta(2)=\emptyset, \quad \Delta(3)=\{0\} .
$$

We only have a vertex $\{0\}$ and two edges from $\{0\}$ to itself, namely $E_{00}=\{0,2\}$. It is clear that the adjacency matrix $B=(2)$ and the Hausdorff dimension

$$
\operatorname{dim}_{H}(T)=\frac{\log 2}{\log 3} .
$$

Example 5.2. Let $A=3$ and $\mathcal{D}=\{0,1,2,5\}$. This is an overlapping system and the attractor $T$ has a nonvoid interior. The dimension is

$$
\operatorname{dim}_{H}(\partial T)=\frac{\log 2}{\log 3} .
$$

Solution. We can choose $\mathcal{C}=\{0,3,6\}$. Then $\Gamma=[0,3] \supset T$. Let $\Gamma_{J}=\psi_{J}(\Gamma)$ for any $J \in \Sigma_{3}$. By the definition we have the following relationship:

The first level:

$$
\begin{aligned}
& \Gamma_{1} \leftrightarrow \Delta(1)=\{0,1,2\}:=v_{1}, \\
& \Gamma_{2} \leftrightarrow \Delta(2)=\{-2,-1,2\}:=v_{2}, \\
& \Gamma_{3} \leftrightarrow \Delta(3)=\{-1\}:=v_{3} .
\end{aligned}
$$

The second level:

$$
\begin{aligned}
& \Gamma_{11} \leftrightarrow \Delta(11)=\{0,1,2\}=v_{1}, \\
& \Gamma_{12} \leftrightarrow \Delta(12)=\{-2,-1,0,1,2\}:=v_{4}, \\
& \Gamma_{13} \leftrightarrow \Delta(13)=\{-2,-1,0,1,2\}:=v_{4} ;
\end{aligned}
$$




$$
\begin{aligned}
& \Gamma_{21} \leftrightarrow \Delta(21)=\{-2,-1,2\}=v_{2}, \\
& \Gamma_{22} \leftrightarrow \Delta(22)=\{-1\}=v_{3}, \\
& \Gamma_{23} \leftrightarrow \Delta(23)=\{0,1,2\}=v_{1} ; \\
& \Gamma_{31} \leftrightarrow \Delta(31)=\{-2,-1\}:=v_{5}, \\
& \Gamma_{32} \leftrightarrow \Delta(32)=\{-1\}=v_{3}, \\
& \Gamma_{33} \leftrightarrow \Delta(33)=\emptyset .
\end{aligned}
$$

The third level:

$$
\begin{aligned}
& \Gamma_{121} \leftrightarrow \Delta(121)=\{-2,-1,0,1,2\}=v_{4}, \\
& \Gamma_{122} \leftrightarrow \Delta(122)=\{-2,-1,0,1,2\}=v_{4}, \\
& \Gamma_{123} \leftrightarrow \Delta(123)=\{-2,-1,0,1,2\}=v_{4} ; \\
& \Gamma_{311} \leftrightarrow \Delta(311)=\{-2,-1,2\}=v_{2}, \\
& \Gamma_{312} \leftrightarrow \Delta(312)=\{-1\}=v_{3}, \\
& \Gamma_{313} \leftrightarrow \Delta(313)=\{0,1,2\}=v_{1} .
\end{aligned}
$$

Let $v_{0}=\{0\}$. From the above we have the following graph-directed relationship:

$$
\begin{array}{lll}
v_{0} \rightarrow v_{1} v_{2} v_{3}, & v_{1} \rightarrow v_{1} v_{4} v_{4}, & \\
v_{3} \rightarrow v_{5} v_{3}, & v_{4} \rightarrow v_{4} v_{4} v_{4}, & v_{5} v_{3} v_{1},
\end{array}
$$

It is not difficult to see that the indices 1 and 12 corresponding to $v_{1}$ and $v_{4}$, respectively, are contained by $\mathcal{S}^{0}$. We discard them and form the new set of vertices $\left\{v_{0}, v_{2}, v_{3}, v_{5}\right\}$ with the graph relationship

$$
\begin{array}{ll}
v_{0} \rightarrow v_{2} v_{3}, & v_{2} \rightarrow v_{2} v_{3}, \\
v_{3} \rightarrow v_{3} v_{5}, & v_{5} \rightarrow v_{2} v_{3} .
\end{array}
$$

Then the adjacency matrix is

$$
B=\left(\begin{array}{llll}
0 & 1 & 1 & 0 \\
0 & 1 & 1 & 0 \\
0 & 0 & 1 & 1 \\
0 & 1 & 1 & 0
\end{array}\right) \text {. }
$$

The spectral radius is $\gamma_{B}=2$ and hence $\operatorname{dim}_{H}(\partial T)=\log 2 / \log 3$ by Theorem 4.3.

Example 5.3. Let $A=3$ and $\mathcal{D}=\{0,1,5\}$. Then

$$
\operatorname{dim}_{H}(\partial T)=\frac{\log \left(3+2^{3 / 2}\right)}{\log 9} \approx 0.802261 .
$$


Solution. If we choose $\mathcal{C}=\{0,3,6\}$ and $\Gamma=[0,3]$ as in the previous example to find the $\Delta(J)$, we need to use six levels of iteration. However, if we perform the following modification, we only need to use three levels.

Note that $T=T(A, \mathcal{D})=T\left(A^{2}, \mathcal{D}_{2}\right)$ with $\mathcal{D}_{2}=\{0,1,3,4,5,8,15,16,20\}$. We choose $\mathcal{C}=\{0,3,6,9,12,15,18,21,24\}$ and $\Gamma=[0,3]$. Similar to the previous example, using $\mathcal{D}_{2}$ instead of $\mathcal{D}$, we get 13 different symbols $\Delta(J)$ for $\mathcal{S}$. Among these symbols two of them correspond to the indices in $\mathcal{S}^{\circ}$. We omit them and build a subgraph-directed relationship for $\partial T$. The adjacency matrix is

$$
B=\left(\begin{array}{lllllllllll}
0 & 1 & 1 & 1 & 2 & 1 & 1 & 0 & 0 & 0 & 0 \\
0 & 2 & 1 & 1 & 0 & 0 & 0 & 1 & 1 & 1 & 1 \\
0 & 0 & 0 & 0 & 0 & 0 & 0 & 1 & 1 & 2 & 0 \\
0 & 1 & 1 & 2 & 1 & 1 & 1 & 0 & 0 & 0 & 1 \\
0 & 0 & 0 & 0 & 2 & 1 & 1 & 0 & 0 & 0 & 0 \\
0 & 1 & 1 & 1 & 0 & 1 & 0 & 1 & 1 & 1 & 1 \\
0 & 1 & 1 & 1 & 1 & 0 & 1 & 0 & 0 & 0 & 0 \\
0 & 1 & 1 & 1 & 1 & 1 & 1 & 1 & 0 & 0 & 1 \\
0 & 1 & 0 & 1 & 0 & 0 & 0 & 0 & 1 & 1 & 1 \\
0 & 0 & 0 & 0 & 0 & 0 & 0 & 1 & 1 & 2 & 0 \\
0 & 0 & 0 & 0 & 2 & 1 & 1 & 0 & 0 & 0 & 0
\end{array}\right) .
$$

Using a computer we get $\gamma_{B}=3+2^{3 / 2}$. Note that the dilation matrix is $A^{2}=9$. Hence, by Theorem 4.3,

$$
\operatorname{dim}_{H}(\partial T)=\frac{\log \left(3+2^{3 / 2}\right)}{\log 9} \approx 0.802261 .
$$

Next we consider an example in the plane. To simplify calculation we use the following lemma for the digit sets having certain symmetry:

Lemma 5.4. Let $A \in M_{n}(\mathbb{Z})$ be an expanding matrix and let $q=|\operatorname{det}(A)|$. Let $\mathcal{D}=$ $-\mathcal{D}$, and let $\mathcal{C}=-\mathcal{C}$, i.e., $c_{i}=-c_{q-i+1}, i=1, \ldots$, q. If $\Delta(J)=-\Delta\left(J^{\prime}\right)$, then

$$
\Delta(J i)=-\Delta\left(J^{\prime} i^{\prime}\right), \quad i=1, \ldots, q,
$$

where $i^{\prime}=q-i+1$.

We omit the proof since it is similar to the proof of Proposition 3.1.

Example 5.5. Let $A=\left(\begin{array}{cc}1 & -1 \\ 1 & 1\end{array}\right)$ and $\mathcal{D}=\left\{(-1,0)^{t},(0,0)^{t},(1,0)^{t}\right\}$. Then

$$
\operatorname{dim}_{H}(\partial T) \approx 1.06924
$$

( $T$ is shown in Figure 1.) 


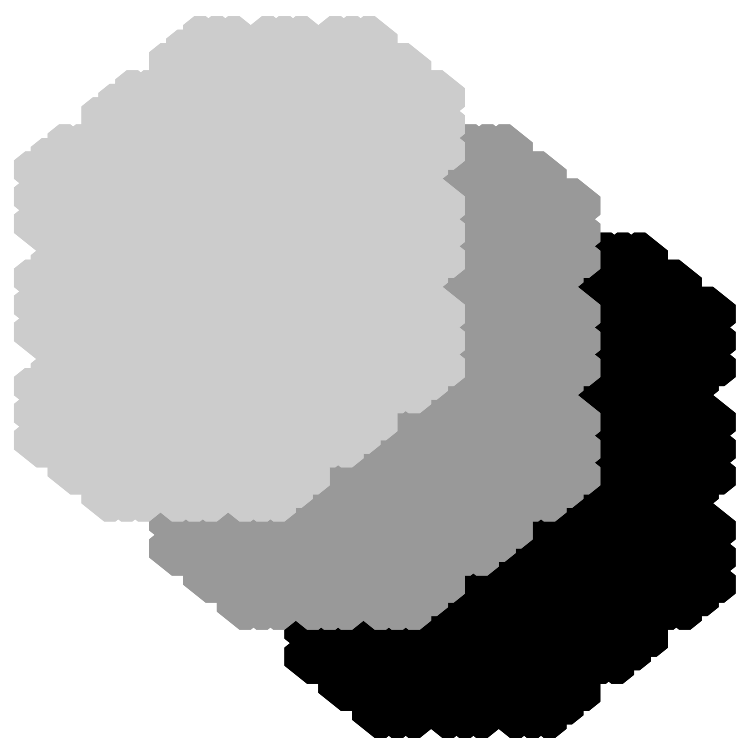

Fig. 1. The self-affine set in Example 5.5.

Solution. A direct application of the algorithm is more complicated and will be discussed in next section. Instead, we will use a similar technique as in the previous example. Since

$$
T=T(A, \mathcal{D})=T\left(A^{4}, \mathcal{D}_{4}\right)=T\left(-4 E, \mathcal{D}_{4}\right)=T\left(4 E, \mathcal{D}_{4}\right),
$$

where the matrix $E$ is the $2 \times 2$ identity matrix. In this case the dilation matrix is $4 E$, let

$$
\begin{aligned}
\mathcal{C}= & \left\{(-6,-6)^{t},(-6,-2)^{t},(-6,2)^{t},(-6,6)^{t},(-2,-6)^{t},(-2,-2)^{t},(-2,2)^{t},\right. \\
& \left.(-2,6)^{t},(2,-6)^{t},(2,-2)^{t},(2,2)^{t},(2,6)^{t},(6,-6)^{t},(6,-2)^{t},(6,2)^{t},(6,6)^{t}\right\} .
\end{aligned}
$$

Then $\Gamma=\{(x, y):-2 \leq x, y \leq 2\}=T(4 E, \mathcal{C})$. It is not difficult to check that $T \subseteq \Gamma^{o}$ and $\Gamma+\mathcal{D} \subseteq 4 \Gamma$. Using the above method and Lemma 5.4, we can get a $35 \times 35$ adjacency matrix and by Theorem $4.3, \operatorname{dim}_{H}(\partial T) \approx 1.06924$.

Note that the twin dragon (see Figure 3 ) is generated by the same $A$ with $\mathcal{D}=$ $\left\{(0,0)^{t},(1,0)^{t}\right\}$ and it is known that its dimension is $\approx 1.523627$. The above self-similar region is generated by one additional digit. It fits the intuition that the boundary is smoother that the twin dragon.

Example 5.6. Let $A=\left(\begin{array}{ll}3 & 0 \\ 0 & 2\end{array}\right)$ and $\mathcal{D}=\left\{(0,0)^{t},(0,1)^{t},(0,2)^{t},(4,0)^{t}\right\}$, then $\operatorname{dim}_{H}$ $(T) \approx 1.54694$. ( $T$ is shown on the right of Figure 2.) 

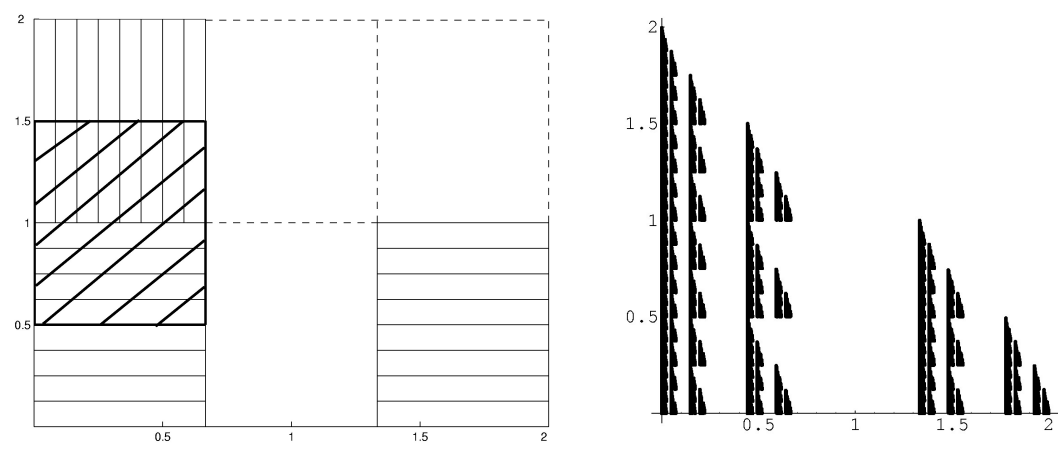

Fig. 2. The overlaps and the self-affine set in Example 5.6.

Solution. Observe that the IFS $\left\{\varphi_{i}(x)=A^{-1}\left(x+d_{i}\right): i=1,2,3,4\right\}$ is overlapping as shown on the left of Figure 2. Let

$$
\mathcal{C}=\left\{(0,0)^{t},(2,0)^{t},(4,0)^{t},(0,2)^{t},(2,2)^{t},(4,2)^{t}\right\}
$$

then $\Gamma=T(A, \mathcal{C})=[0,2]^{2}$ and $\Gamma+\mathcal{D} \subseteq A \Gamma$. Denote $\Gamma_{J}=\psi_{J}(X)$ for $J \in \Sigma_{6}$. The vertices are constructed as the following:

The zeroth level is $\Gamma \leftrightarrow \Delta(0)=\left\{(0,0)^{t}\right\}:=v_{0}$.

The first level:

$$
\begin{aligned}
& \Gamma_{1} \leftrightarrow \Delta(1)=\left\{(0,0)^{t},(0,1)^{t}\right\}:=v_{1}, \quad \Gamma_{4} \leftrightarrow \Delta(4)=\left\{(0,-1)^{t},(0,0)^{t}\right\}:=v_{2}, \\
& \Gamma_{2} \leftrightarrow \Delta(2)=\emptyset \text {, } \\
& \Gamma_{5} \leftrightarrow \Delta(5)=\emptyset, \\
& \Gamma_{3} \leftrightarrow \Delta(3)=\left\{(0,0)^{t}\right\}=v_{0}, \quad \Gamma_{6} \leftrightarrow \Delta(6)=\emptyset .
\end{aligned}
$$

The second level:

$$
\begin{aligned}
& \Gamma_{11} \leftrightarrow \Delta(11)=v_{1}, \quad \Gamma_{14} \leftrightarrow \Delta(14)=\left\{(0,0)^{t},(0,1)^{t},(0,-1)^{t}\right\}:=v_{3}, \\
& \Gamma_{12} \leftrightarrow \Delta(12)=\emptyset, \quad \Gamma_{15} \leftrightarrow \Delta(5)=\emptyset, \\
& \Gamma_{13} \leftrightarrow \Delta(13)=v_{0}, \quad \Gamma_{16} \leftrightarrow \Delta(16)=v_{0} ; \\
& \Gamma_{41} \leftrightarrow \Delta(41)=v_{3}, \quad \Gamma_{44} \leftrightarrow \Delta(44)=v_{2}, \\
& \Gamma_{42} \leftrightarrow \Delta(42)=\emptyset, \quad \Gamma_{45} \leftrightarrow \Delta(45)=\emptyset, \\
& \Gamma_{43} \leftrightarrow \Delta(43)=v_{0}, \quad \Gamma_{46} \leftrightarrow \Delta(46)=\emptyset .
\end{aligned}
$$

The third level:

$$
\begin{aligned}
& \Gamma_{141} \leftrightarrow \Delta(141)=v_{3}, \quad \Gamma_{144} \leftrightarrow \Delta(144)=v_{3}, \\
& \Gamma_{142} \leftrightarrow \Delta(142)=\emptyset, \quad \Gamma_{145} \leftrightarrow \Delta(145)=\emptyset, \\
& \Gamma_{143} \leftrightarrow \Delta(143)=v_{0}, \quad \Gamma_{146} \leftrightarrow \Delta(146)=v_{0} .
\end{aligned}
$$

Let us represent the iteration $\Gamma_{J}$ to its descendents $\Gamma_{J i}, 1 \leq i \leq 6$, by

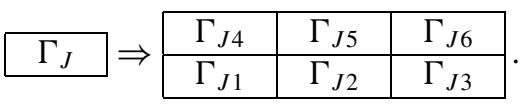


The corresponding relationships are

\begin{tabular}{|c|c|c|c|c|c|c|c|c|c|}
\hline \multirow[b]{2}{*}{$v_{0}$} & \multirow{2}{*}{$\Rightarrow$} & $v_{2}$ & $\varnothing$ & $\varnothing$ & \multirow[b]{2}{*}{$v_{1}$} & \multirow[b]{2}{*}{$\Rightarrow$} & $v_{3}$ & \multirow{2}{*}{$\frac{\emptyset}{\emptyset}$} & \multirow{2}{*}{$\frac{v_{0}}{v_{0}}$} \\
\hline & & $v_{1}$ & $\varnothing$ & $v_{0}$ & & & $v_{1}$ & & \\
\hline \multirow[b]{2}{*}{$v_{2}$} & \multirow[b]{2}{*}{$\Rightarrow$} & $v_{2}$ & $\varnothing$ & $\emptyset$ & \multirow[b]{2}{*}{$v_{3}$} & \multirow{2}{*}{$\Rightarrow$} & $v_{3}$ & $\varnothing$ & $v_{0}$ \\
\hline & & $v_{3}$ & $\varnothing$ & $v_{0}$ & & & $v_{3}$ & $\emptyset$ & $v_{0}$ \\
\hline
\end{tabular}

Let

$$
B_{0}=\left(\begin{array}{llll}
1 & 1 & 0 & 0 \\
1 & 1 & 0 & 0 \\
1 & 0 & 0 & 1 \\
1 & 0 & 0 & 1
\end{array}\right), \quad B_{1}=\left(\begin{array}{llll}
0 & 0 & 1 & 0 \\
1 & 0 & 0 & 1 \\
1 & 0 & 0 & 0 \\
1 & 0 & 0 & 1
\end{array}\right)
$$

be the adjacency matrices corresponding to the edges for the lower and upper rows, respectively. Then by Theorem 4.4 we can use an approximate calculation by Mathematica to show that $\operatorname{dim}_{H}(T) \approx 1.54694$.

\section{Remarks on the Algorithm}

For the above examples we have very simple ways of constructing the auxiliary tiles with simple geometry. However, in general, the geometry of a tile is not clear. This creates problems in finding the $\Delta(J)$ 's because we have to determine

$$
\varphi_{I}(\Gamma) \cap \psi_{J}(\Gamma) \neq \varnothing .
$$

A method to get by this difficulty is to introduce a "seed set" $X$ of simple geometry (e.g., a large ball) with

$$
\Gamma \subseteq X \quad \text { and } \quad \varphi_{i}(X) \subseteq X, \quad \psi_{j}(X) \subseteq X, \quad 1 \leq i \leq N, \quad 1 \leq j \leq q .
$$

We redefine the symbols $\Delta(J)$ by

$$
\Delta(J)=\left\{d_{I}-c_{J}: I \in \Sigma_{N}^{k}, \varphi_{I}(X) \cap \psi_{J}(X) \neq \emptyset\right\} .
$$

There is, in fact, no essential change in replacing $\Gamma$ with $X$ in Proposition 2.1, Theorem 2.2, and Proposition 3.1, and their proofs (in the proof of Proposition 3.1 the hypothesis $\Gamma+\mathcal{D} \subseteq A \Gamma$ in (A1) is replaced by (6.1)). The only price to pay for this new $X$ is to make the graph-directed system larger.

The bigger problem is to construct the tile for the seemingly obvious condition $T \subset \Gamma^{o}$ (or $T \subseteq \Gamma$ ), since the self-affine set $T$ and the auxiliary tile $\Gamma$ is of unknown geometry and it is not so obvious to select the $\operatorname{digit} \operatorname{set} \mathcal{C}$ so that $T \subseteq \Gamma^{o}$. In the following we will describe how this can be done in general. First we prove a lemma:

Lemma 6.1. Let $A \in M_{n}(\mathbb{Z})$ be an expanding matrix and let $\mathcal{C} \in \mathbb{Z}^{n}$ be a digit set with $0 \in \mathcal{C}$. Let $\|\cdot\|$ be a norm such that $\left\|A^{-1}\right\|<1$ and let $\eta \geq\left\|A^{-1}\right\|\left(\max _{i}\left\|c_{i}\right\|\right) /(1-$ $\left.\left\|A^{-1}\right\|\right)$. Then there exists a $k$ such that

$$
\left(\mathcal{C}_{k+1} \backslash \mathcal{C}_{k}\right) \cap B_{\eta}=\emptyset .
$$

This implies that $\left(\mathcal{C}_{m} \backslash \mathcal{C}_{k}\right) \cap B_{\eta}=\emptyset$ for all $m>k$. 
Proof. For any $\alpha \in \mathcal{C}_{k+2} \backslash \mathcal{C}_{k}$, we need to prove $\|\alpha\|>\eta$. If $\alpha \in \mathcal{C}_{k+1}$, nothing need be done. If $\alpha \notin \mathcal{C}_{k+1}$, we have $\alpha=A \alpha^{\prime}+c_{i}$ for some $\alpha^{\prime} \in \mathcal{C}_{k+1} \backslash \mathcal{C}_{k}$ and $c_{i} \in \mathcal{C}$. It follows that

$$
\left\|\alpha^{\prime}\right\| \leq\left\|A^{-1}\right\|\|\alpha\|+\left\|A^{-1}\right\|\left\|c_{i}\right\| \leq \eta .
$$

This contradicts the hypothesis $\left(\mathcal{C}_{k+1} \backslash \mathcal{C}_{k}\right) \cap B_{\eta}=\emptyset$. Hence $\left(\mathcal{C}_{k+2} \backslash \mathcal{C}_{k}\right) \cap B_{\eta}=\emptyset$. By induction the result follows.

Now, to choose the desired auxiliary tile, we pick a complete set of coset representation $\mathcal{G}=\left\{0=g_{1}, \ldots, g_{q}\right\}$ of $\mathbb{Z}^{n} / A \mathbb{Z}^{n}$. Then $G=T(A, \mathcal{G})$ is a self-affine tile [19]. We use the idea described in Application IV (with the above modification of $X$, if necessary) to find all the $\Delta(J)$ and choose a maximal $\Delta(J), J=\left(j_{1}, \ldots, j_{l}\right)$. Then $G_{J}$ is in the interior of $G$ by Theorem 4.6. The point $x_{0}=A^{-1} g_{j_{1}}+\cdots+A^{-l} g_{j_{l}}$ is hence an interior point.

Let

$$
\tilde{G}=G-x_{0} \quad \text { and } \quad \tilde{\mathcal{G}}=\mathcal{G}_{l}-A^{l} x_{0}
$$

(here $\mathcal{G}_{l}=\mathcal{G}+A \mathcal{G}+\cdots+A^{l-1} \mathcal{G}$ as defined previously). Then $0 \in \tilde{G}$ and $A^{l} \tilde{G}=\tilde{G}+\tilde{\mathcal{G}}$ with $0 \in \tilde{\mathcal{G}}$, i.e., $\tilde{G}$ is a tile with respect to the matrix $A^{l}$ and $\operatorname{digit}$ set $\tilde{\mathcal{G}}$. Let

$$
\eta \geq \max \left\{\frac{\left\|A^{-l}\right\| \max _{i}\left\|\tilde{g}_{i}\right\|}{1-\left\|A^{-l}\right\|}, t\right\},
$$

where $t \geq 2|T|$ and can be determined a priori. By using the tiling property and the $k$ in the lemma, we see that if we let

$$
\Gamma=A^{l k}(\tilde{G})=\tilde{G}+\tilde{\mathcal{G}}_{k},
$$

then $\Gamma$ contains $B_{\eta}$ and hence $T \subset \Gamma^{o}$. This is a direct check that $A \Gamma=\Gamma+\mathcal{C}$ where $\mathcal{C}=A^{l k}\left(\mathcal{G}+x_{0}-A x_{0}\right) \subseteq \mathbb{Z}^{n}$ is the digit set.

To illustrate the above remarks we will look at Example 5.5 again

$$
A=\left(\begin{array}{rr}
1 & -1 \\
1 & 1
\end{array}\right) \quad \text { and } \quad \mathcal{D}=\left\{(-1,0)^{t},(0,0)^{t},(1,0)^{t}\right\}
$$

We start with the twin dragon with digits $\mathcal{G}=\left\{(0,0)^{t},(1,0)^{t}\right\}$. We can use the algorithm described above to find an interior point $x_{0}$. For this special case we can show directly that

$$
x_{0}=\left(\frac{1}{4},-\frac{1}{2}\right)^{t}=A^{-1}(1,0)^{t}+A^{-4}(1,0)^{t} \in G,
$$

but

$$
x_{0} \notin G+\left\{(1,0)^{t},(-1,0)^{t},(0,1)^{t},(0,-1)^{t},(1,-1)^{t},(-1,1)^{t}\right\},
$$

i.e., $x_{0}$ is not in the six neighboring tiles. Hence $x_{0} \in G^{o}$. (As a note we observe that the origin is not in the interior of $G$. Indeed, $(0,-1)^{t}=\sum_{i=1}^{\infty} A^{-i}(1,0)^{t}$ implies that $(0,0)^{t} \in G+(0,1)^{t}$. Since $\mathbb{Z}^{2}$ is a tiling set of $T$ and $(0,0)^{t} \in G \cap\left(G+(0,1)^{t}\right)$, it follows that $(0,0)^{t}$ is in the boundary of $T$.) 


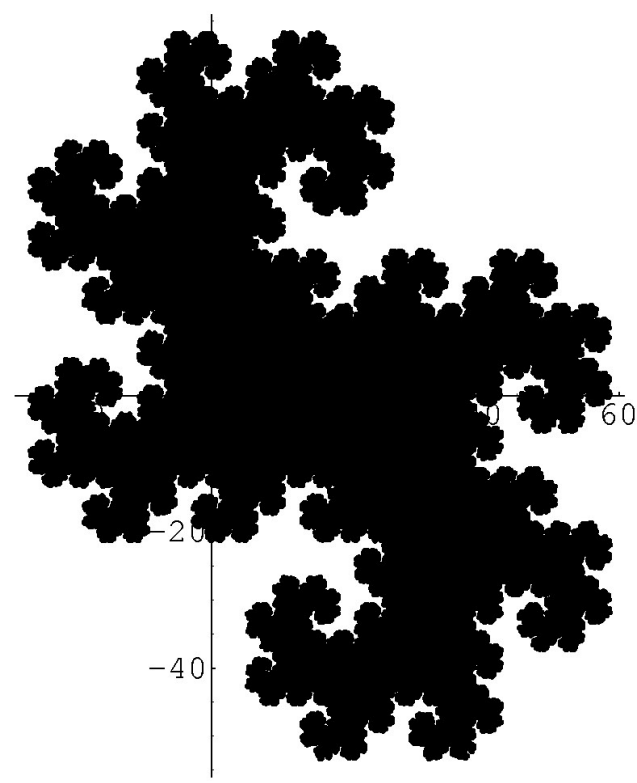

Fig. 3. The self-affine tile $\Gamma$

We let $\tilde{G}=G-x_{0}$. By the above estimate we have that $T \subseteq A^{12}(\tilde{G})$. Hence we can take $\mathcal{C}=\left\{(32,16)^{t},(-32,16)^{t}\right\}$ and $\Gamma=A^{12}(\tilde{G})$. This completes the choice of the tile. The picture of $\Gamma$ is shown in Figure 3.

Note that it is not easy to check whether $\Gamma+\mathcal{D} \subseteq A \Gamma$. We hence select $X=B_{r}(0)$, a ball with $r=16(\sqrt{10}+\sqrt{5})$, then $\Gamma \subseteq X$ and (6.1) holds. $X$ is used as the seed set. Using the algorithm we have a $52 \times 52$ adjacency matrix. By Theorem 4.3 we can calculate the Hausdorff dimension of $\partial T$ approximate to $\approx 1.06924$, the same as in Example 5.5.

Acknowledgments. The authors like to thank K. S. Leung for some helpful advice in preparing the illustrations. They are also grateful to the referees for their valuable comments and suggestions, in particular, for pointing out the preprint of Dekking and Van Der Wal [6] which deals with the same problem but using a complete different method, the cellular automata instead of the geometric approach here. The research is partially supported by an HK RGC grant and a direct grant from CUHK.

\section{References}

1. C. BANDT (1991): Self-similar sets 5. Integer matrices and fractal tilings of $\mathbb{R}^{n}$. Proc. Amer. Math. Soc., 112:549-562.

2. T. BEDFORD (1984): Crinkly curves, Markov partitions and box dimension in self-similar sets. Ph.D. Thesis. University of Warwick.

3. I. DAUBECHIES (1992): Ten Lecture on Wavelets. Philadelphia, PA: SIAM.

4. I. DAUBECHIES, J. C. LAGARIAS (1992): Two-scale difference equations II: Infinite matrix products, local regularity and fractals. SIAM J. Appl. Math., 23:1031-1079.

5. F. M. DEKKING (1982): Recurrent sets. Adv. in Math. 44:78-104.

6. F. M. DEKKING, P. VAN DER WAL (Preprint): The boundary of an attractor of a recurrent iterated function system. 
7. P. Duvall, J. KeESLING, A. VINCE (2000): The Hausdorff dimension of the boundary of a self-similar tile. J. London Math. Soc. (2), 61:748-760.

8. K. J. FALCONER (1990): Fractal Geometry: Mathematical Foundations and Applications. New York: Wiley.

9. K. J. FALCONER (1997): Techniques in Fractal Geometry. Chichester: Wiley.

10. K. GRÖCHENIG, A. HAAS (1994): Self-similar lattice tilings. J. Fourier Anal. Appl., 1:131-170.

11. K. GRÖCHEnIG, A. HAAS, A. RAUGI (1999): Self-affine tilings with several tiles. I. Appl. Comput. Harmonic Anal., 7:211-238.

12. K. GRÖCHENIG, W. R. MADYCH (1992): Multiresolution analysis, Haar bases, and self-similar tilings of $R^{n}$. IEEE Trans. Inform. Theory, 38:556-568.

13. X.-G. HE (2001): The geometry of self-affine sets and graph-directed systems. Ph.D. Thesis. The Chinese University of Hong Kong.

14. J. E. HUTCHINSON (1981): Fractals and self-similarity. Indiana Univ. Math. J., 30:713-747.

15. R. Q. JIA (1995): Subdivision schemes in $L_{p}$ spaces. Adv. in Comput. Math., 3:309-341.

16. R. KENYON (1992): Self-replicating tilings. In: Symbolic Dynamics and Its Applications (P. Walters, ed.). Contemporary Mathematicians, Vol. 135. Boston: Birkhäuser, pp. 239-264.

17. R. KENYON, Y. PERES (1996): Hausdorff dimensions of sofic affine-invariant sets. Israel J. Math., 94:157-178.

18. I. KIRAT, K.-S. LAU (2000): On the connectedness of self-affine tiles. J. London Math. Soc. (2), 62:291304.

19. J. C. LaGaRIAS, Y. WANG (1996): Self-affine tiles in $\mathbb{R}^{n}$. Adv. in Math., 121:21-49.

20. J. C. LAGARIAS, Y. WANG (1996): Integral self-affine tiles in $\mathbb{R}^{n}$, Part I. Standard and non-standard digit sets. J. London Math. Soc., 53:21-49.

21. J. C. LAGARIAS, Y. WANG (1997): Integral self-affine tiles in $\mathbb{R}^{n}$, Part II. Lattice tilings. J. Fourier Anal. Appl., 3:84-102.

22. S. P. LALley (1997): $\beta$-Expansions with deleted digits for Pisot numbers. Trans. Amer. Math. Soc., 349:4355-4365.

23. K.-S. LAU, S.-M. NGAI, H. RAO (2001): Iterated function systems with overlaps and the self-similar measures. J. London Math. Soc., 63:99-115.

24. R. D. MAULDIN, S. C. WILLIAMS (1988): Hausdorff dimension in graph directed constructions. Trans. Amer. Math. Soc., 309:811-829.

25. C. MCMULLEN (1984): The Hausdorff dimension of general Sierpinski carpets. Nagoya Math. J., 96:1-9.

26. S.-M. NGAI, Y. WANG (2001): Hausdorff dimension of overlapping self-similar sets. J. London Math. Soc. (2), 63:655-672.

27. A. M. ODLYZKO (1978): Non-negative digit sets in positional number systems. Proc. London Math. Soc. (3), 37:213-229.

28. H. RAO, Z.-Y. WEN (1998): Some studies of a class of self-similar fractals with overlap structure. Adv. in Appl. Math., 20:50-72.

29. E. SENETA (1981): Non-Negative Matrixes and Markov Chains. New York: Springer-Verlag.

30. R. S. STRICHARTZ, Y. WANG (1999): Geometry of self-affine tiles. I. Indiana Univ. Math. J., 48:1-23.

31. J. J. P. VEeRmAn (1998): Hausdorff dimension of boundaries of self-affine tiles in $\mathbf{R}^{N}$. Bol. Soc. Mat. Mexicana (3), 4:159-182.

32. Y. WANG (1999): Self-affine tiles. In: Advances in Wavelets (K.-S. Lau, ed.). Singapore: Springer-Verlag, pp. 261-282.

33. M. P. W. ZERNER (1996): Weak separation properties for self-similar sets. Proc. Amer. Math. Soc., 124:3529-3539.

34. D. X. ZHOU (2001): Self-similar lattice tilings and subdivision schemes. SIAM J. Math. Anal., 33:1-15.

\section{X.-G. He}

Department of Mathematics

The Chinese University of

Hong Kong

Shatin

Hong Kong

xghe@sina.com

K.-S. Lau
Department of Mathematics
The Chinese University of
Hong Kong
Shatin
Hong Kong
kslau@math.cuhk.edu.hk

H. Rao

Department of Mathematics

Wuhan University

Wuhan

P. R. China

raohui@nlsc.whu.edu.cn 